EPJ Web of Conferences 52, 01005 (2013)

DOI: 10.1051/epjconf/20135201005

C Owned by the authors, published by EDP Sciences, 2013

\title{
The LHCb experiment: status and recent results
}

\author{
Dmytro Volyanskyy (on behalf of the LHCb collaboration) \\ ${ }^{1}$ Max-Planck-Institut für Kernphysik, PO Box 103980, 69029 Heidelberg, Germany
}

\begin{abstract}
The LHCb experiment is one of the major research projects at the Large Hadron Collider. Its acceptance and instrumentation is optimised to perform high-precision studies of flavour physics and particle production in a unique kinematic range at unprecedented collision energies. Using large data samples accumulated in the years 2010-2012, the LHCb collaboration has conducted a series of measurements providing a sensitive test of the Standard Model and strengthening our knowledge of flavour physics, QCD and electroweak processes. The status of the experiment and some of its recent results are presented here.
\end{abstract}

\section{The LHCb experiment}

Due to its unprecedented interaction rate and collision energy, the Large Hadron Collider (LHC) delivers a vast amount of heavy flavour particles. This provides an excellent opportunity to perform high-precision measurements and explore rare processes in the heavy flavour sector. The Large Hadron Collider beauty (LHCb) experiment has been mainly designed to study the physics of the heaviest hadrons - the beauty flavoured ones ( $B$ hadrons). In particular, the experiment aims to explore $C P$-violating phenomena in the $B$ hadron sector and highly suppressed flavour-changing neutral currents (FCNC) using appropriate $B$ hadron decay channels plus overconstrain the CKM unitary triangle. These measurements should provide a rigorous test of the Standard Model while indirectly probing for New Physics effects. The latter may appear as contributions from new virtual heavy particles in loopmediated processes giving access to scales greater than the LHC centre-of-mass energy. Another important goal of the experiment is to fulfil a broad charm physics programme.

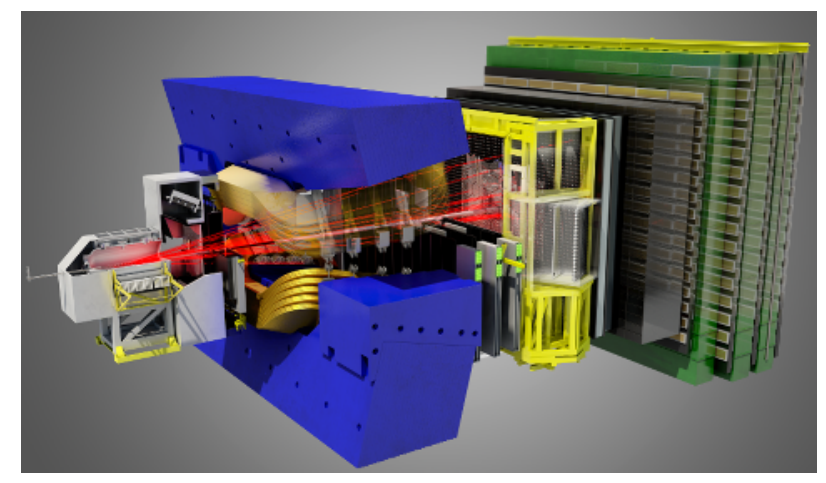

Figure 1: View of the LHCb spectrometer.
Since $B$ hadrons at the LHC are mainly produced at low polar angle in the same forward cone, the $\mathrm{LHCb}$ detector [1] has been constructed as a single-arm, forward spectrometer, as can be seen in Fig. 1. Its angular coverage ranges from $10 \mathrm{mrad}$ to 300 (250) mrad in the bending (non-bending) plane allowing particle reconstruction in the pseudorapidity range $2<\eta<5$. Although the spectrometer covers just about $4 \%$ of the solid angle, it detects about $40 \%$ of heavy quark hadrons produced at the LHC. Due to its unique pseudorapidity coverage and the ability to perform measurements at low transverse momenta $p_{\mathrm{T}}$, the detector also permits a unique insight into particle production in the forward region at the LHC.

\section{Detector performance and data taking in 2009-2012}

The LHCb subcomponents can be split into the following two categories: tracking detectors, which are used to reconstruct the trajectories and momenta of charged particles, and particle identification detectors, which are employed to identify different types of particles. The $\mathrm{LHCb}$ tracking system covers the full acceptance of the experiment which is unique at the LHC. It consists of a siliconstrip vertex detector (VELO) surrounding the collision region, a large-area silicon-strip detector located upstream of a dipole magnet with a bending power of about $4 \mathrm{Tm}$, and three stations of silicon-strip detectors and straw drift tubes placed downstream. The VELO has a larger angular acceptance than the rest of the spectrometer, including partial coverage of the backward region which allows reconstruction of charged particle tracks in the pseudorapidity ranges $1.5<\eta<5.0$ and $-4<\eta<-1.5$. Its sensors are positioned along and perpendicular to the beam axis being split into two movable halves. Their sensitive area during stable beam conditions starts at $8 \mathrm{~mm}$ from the beam axis, providing full azimuthal coverage. The VELO measures the radial and azimuthal track coordinates around the lu-

This is an Open Access article distributed under the terms of the Creative Commons Attribution License 2.0, which permits unrestricted use, distribution, and reproduction in any medium, provided the original work is properly cited. 

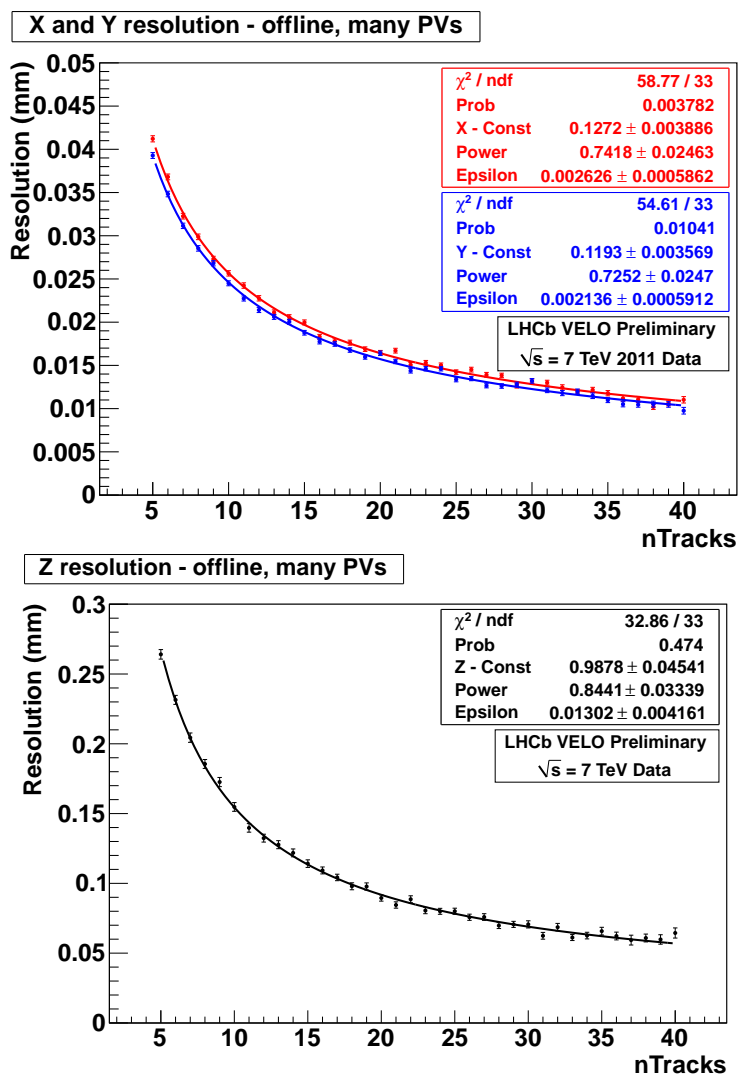

Figure 2: Primary vertex resolution along the $X$ (shown in red), $\mathrm{Y}$ (shown in blue) and $\mathrm{Z}$ (shown in black) axes as a function of the number of tracks used in the vertex fit.

minous region with high precision. In particular, a hit resolution of $4 \mu \mathrm{m}$ is achieved at the minimal pitch and optimal track angle along with a hit finding efficiency over $99 \%$ and an alignment accuracy of $1 \mu \mathrm{m}$. This allows high-precision reconstruction of the positions of the primary and decay vertices. Figure 2 illustrates the achieved primary vertex resolution as a function of the number of tracks used in the vertex fit, while the impact parameter resolution along the $\mathrm{X}$-axis is shown in Fig. 3 as a function of $p_{\mathrm{T}}$. The combined tracking system has a momentum resolution $\Delta p / p$ that varies from $0.4 \%$ at $5 \mathrm{GeV} / c$ to $0.6 \%$ at $100 \mathrm{GeV} / c$, and an impact parameter resolution of about $20 \mu \mathrm{m}$ for tracks with high $p_{\mathrm{T}}$. The overall track finding efficiency is found to exceed $96 \%$ for tracks with momentum greater than $5 \mathrm{GeV} / c$.

An excellent performance of the $\mathrm{LHCb}$ tracking system permits high-precision measurements of the invariant mass of different resonances decaying into charged particles. In particular, the LHCb collaboration has performed the world's most precise measurement of several $B$ hadron masses, which was achieved with just $35 \mathrm{pb}^{-1}$ of data collected in 2010 [2]. Examples of different invariant mass distributions are shown in Fig. 4.

Due to the excellent vertexing capabilities provided by the VELO, the LHCb spectrometer measures the proper decay time of $B$ hadrons with resolution of better than $50 \mathrm{fs}$. This allows a precise resolving of rapid $B_{s}^{0}-\bar{B}_{s}^{0}$

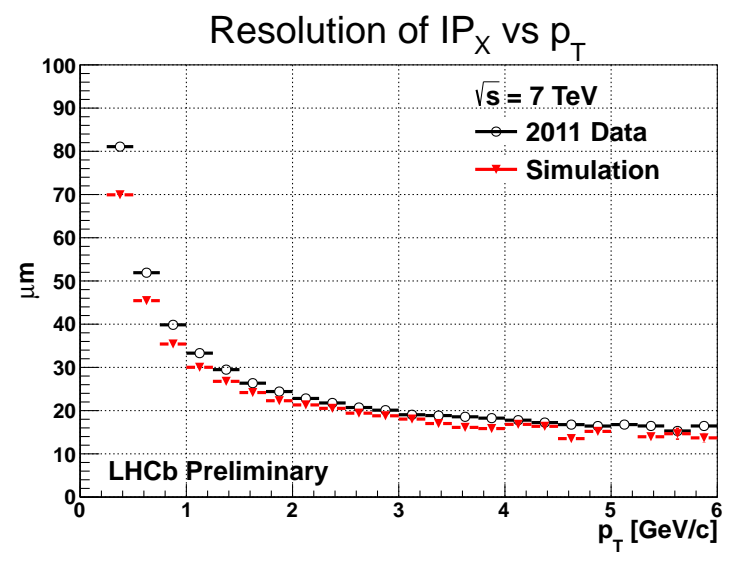

Figure 3: Impact parameter resolution along the $\mathrm{X}$-axis as a function $p_{\mathrm{T}}$.
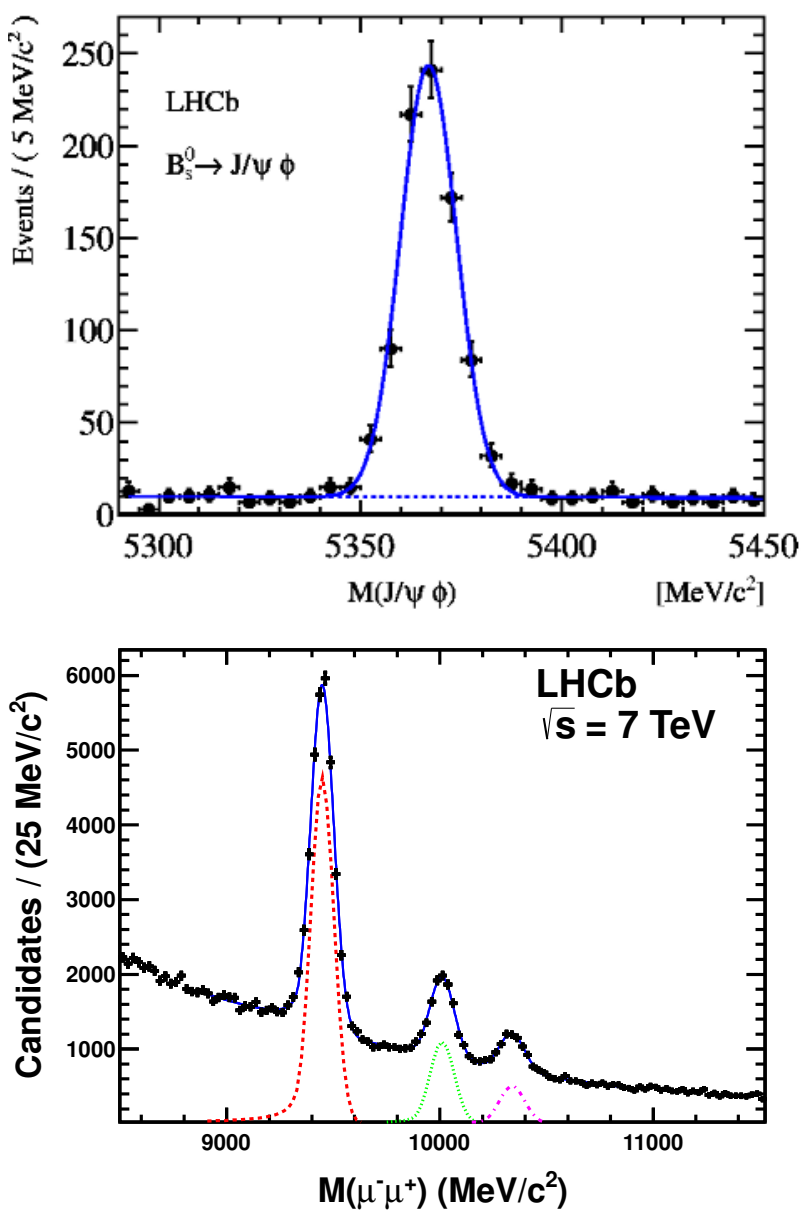

Figure 4: Invariant mass distribution of the $B_{s}^{0}$ meson decaying into $J / \psi \phi$ resonances (top) and $\Upsilon$ mesons decaying into two muons (bottom). The corresponding mass resolutions are $7 \mathrm{MeV} / c^{2}$ for the $B_{s}^{0}$ meson and $54 \mathrm{MeV} / c^{2}$ for the $\Upsilon(1 S)$ meson.

oscillations which is essential for time-dependent mixing and $C P$ violation studies in this system. 

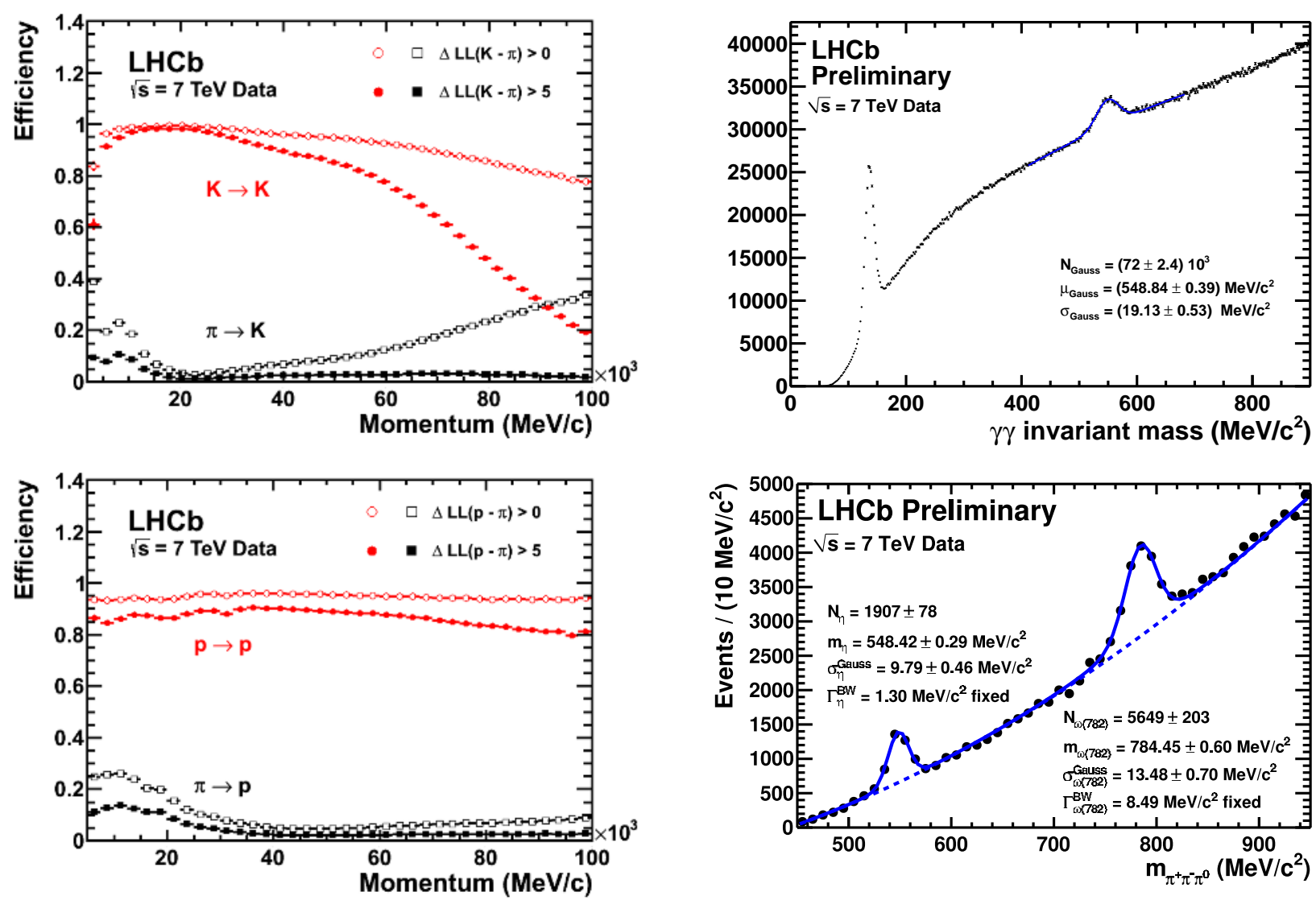

Figure 5: Kaon (top) and proton (bottom) identification efficiency (circles) and pion misidentification rate (squares) as a function of momentum for tight and loose selections.

Robust particle identification plays an important role in the suppression of combinatorial background, separation of decay channels with identical topology and flavour tagging. Charged hadrons at $\mathrm{LHCb}$ are identified using two ring-imaging Cherenkov detectors with three different radiators. These provide efficient $K / \pi$ and $p / \pi$ separations over the momentum range $2<p<100 \mathrm{GeV} / c$. Figure 5 illustrates the efficiency of kaon and proton identification along with the corresponding pion misidentification rate as a function of momentum for tight and loose selection criteria. For the majority of kaons originating from $B$ hadron decays the identification efficiency is found to be above $95 \%$ with a pion misidentification rate below $10 \%$.

Photon, electron and hadron candidates are distinguished by a calorimeter system consisting of scintillating-pad and preshower detectors, an electromagnetic calorimeter (ECAL) and a hadronic calorimeter (HCAL). Electrons and photons are fully absorbed in the ECAL which measures their energies with a resolution of $\sigma(E) / E=10 \% / \sqrt{E} \oplus 1 \%$ (with $E$ in $\mathrm{GeV}$ ). This allows reconstruction of various decay channels involving these particles as illustrated in Fig. 6. The energy resolution of the HCAL is $\sigma(E) / E=69 \% / \sqrt{E} \oplus 9 \%$. In contrast to the ECAL, its information is not directly used in the of-

Figure 6: Diphoton invariant mass distribution (top) with $\pi^{0}$ plus $\eta$ peaks and $\pi^{+} \pi^{-} \pi^{0}(\gamma \gamma)$ invariant mass distribution (bottom) with $\eta$ plus $\omega(782)$ peaks.

fline analyses, while being very important for triggering purposes.

Muon detection is performed by a system positioned downstream of the calorimeters and composed of alternating layers of iron and multiwire proportional chambers. The overall muon identification efficiency exceeds $95 \%$ with a misidentification rate of about $3 \%$ which is mainly due to decays in flight.

The LHCb experiment possesses a selective and flexible trigger system which allows the recording of events with different topologies. It consists of a hardware stage, based on information from the calorimeters and muon systems, followed by a software stage which applies a full event reconstruction. The maximum output rate of the hardware and software stages is currently limited to about $1 \mathrm{MHz}$ and $5 \mathrm{kHz}$, respectively.

First proton-proton $(p p)$ collisions at the $\mathrm{LHCb}$ interaction point occurred in November 2009. Since then and until the end of 2012, the experiment has recorded around $3 \mathrm{fb}^{-1}$ of $p p$ collision data at four different centre-of-mass energies $(\sqrt{s})$ as summarised in Table 1 . Around $99 \%$ of read-out channels were operational throughout the entire data taking period and only $1 \%$ of accumulated data was discarded for physics analyses. To expand its physics programme, the experiment also participates in the protonlead collision data taking. 
Table 1: pp collision data recorded in 2009-2012.

\begin{tabular}{c|c|c} 
Year & $\sqrt{s}(\mathrm{TeV})$ & Data \\
\hline 2009 & 0.9 & $6.8 \mu \mathrm{b}^{-1}$ \\
2010 & 0.9 & $0.3 \mathrm{nb}^{-1}$ \\
2010 & 7.0 & $37 \mathrm{pb}^{-1}$ \\
2011 & 2.76 & $0.1 \mathrm{pb}^{-1}$ \\
2011 & 7.0 & $1.0 \mathrm{fb}^{-1}$ \\
2012 & 8.0 & $2.0 \mathrm{fb}^{-1}$
\end{tabular}

LHCb Efficiency breakdown pp collisions 2010-2012

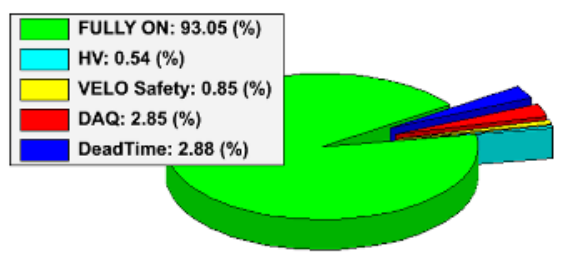

Figure 7: Data taking efficiency breakdown in 2010-2012.

The $\mathrm{LHCb}$ detector was originally designed to run at moderate luminosity as high pile-up environment complicates flavour tagging and identification of $B$ hadron decay vertices. Nevertheless, the majority of the data in 2011 and 2012 were recorded at an instantaneous luminosity nearly a factor of two above the $\mathrm{LHCb}$ design value $\left(L=2.0 \times 10^{32} \mathrm{~cm}^{-2} \mathrm{~s}^{-1}\right)$ and a pile-up rate four times higher than the nominal value $(\mu=0.4)$. This posed a strong challenge to the trigger plus offline data processing and the experiment has demonstrated the ability to successfully cope with these extreme running conditions. Its overall data taking efficiency during the period 2010-2012 exceeded $90 \%$ as can be seen in Fig. 7. To moderate the instantaneous luminosity at the LHCb collision point with respect to the LHC nominal interaction rate in 2011 and 2012, the accelerator was continuously displacing both proton beams to reduce the area of interactions where the proton bunches cross through each other. Due to this technique, called luminosity levelling, the interaction rate at the LHCb collision point was kept at a roughly constant value throughout an entire LHC fill.

\section{Selected physics results}

Using large samples of $p p$ collision data accumulated in 2010-2012, the LHCb collaboration has performed a series of measurements that provides a sensitive test of the Standard Model delivering valuable input to the existing theories. Some of these are summarised hereafter.

\subsection{Rare B hadron decays}

In the Standard Model, the rare leptonic and semileptonic decays $B_{(s)}^{0} \rightarrow \mu^{+} \mu^{-}$and $B^{0} \rightarrow K^{* 0} \mu^{+} \mu^{-}$are highly sup- pressed FCNC processes mediated by electroweak box and penguin type diagrams. These decays have an enhanced sensitivity to physics beyond the Standard Model, as new heavy particles may enter in diagrams, which compete with the Standard Model processes, strongly affecting branching fractions or angular distributions of the daughter particles. Looking for evidence of New Physics, the $\mathrm{LHCb}$ collaboration has recently studied these processes with currently available data. It should be noted that the outcome of these analyses imposes strong constraints on supersymmetric models [3].

\subsection{1 $B_{(s)}^{0} \rightarrow \mu^{+} \mu^{-}$}

A recent search for the rare decays $B_{s}^{0} \rightarrow \mu^{+} \mu^{-}$and $B^{0} \rightarrow \mu^{+} \mu^{-}$is conducted with $1.0 \mathrm{fb}^{-1}$ and $1.1 \mathrm{fb}^{-1}$ of $p p$ collision data recorded at $\sqrt{s}=7 \mathrm{TeV}$ and $\sqrt{s}=8 \mathrm{TeV}$, respectively [4]. Signal candidates are selected among the triggered events by requiring two high quality muon candidates originating from a common vertex, which is well displaced with respect to any $p p$ interaction vertex, and having an invariant mass close to the $B_{(s)}^{0}$ nominal mass. A multivariate selection, based on boosted decision trees [5], is applied at the next stage. It rigorously suppresses the background contamination retaining the majority of signal as determined from simulation.

Taking into account the measured normalisation factors and assuming the Standard Model branching fractions, the combined 2011 and 2012 data sample is expected to contain about $14.1 B_{s}^{0} \rightarrow \mu^{+} \mu^{-}$and $1.7 B^{0} \rightarrow \mu^{+} \mu^{-}$decays. Figure 8 illustrates the resulting invariant mass distribution of the selected $B_{s}^{0} \rightarrow \mu^{+} \mu^{-}$candidates, where the signal shape is described by a Crystal Ball function. The central peak values for the $B_{s}^{0}$ and $B^{0}$ mesons are obtained from the control channels $B_{s}^{0} \rightarrow K^{+} K^{-}, B^{0} \rightarrow K^{+} \pi^{-}$

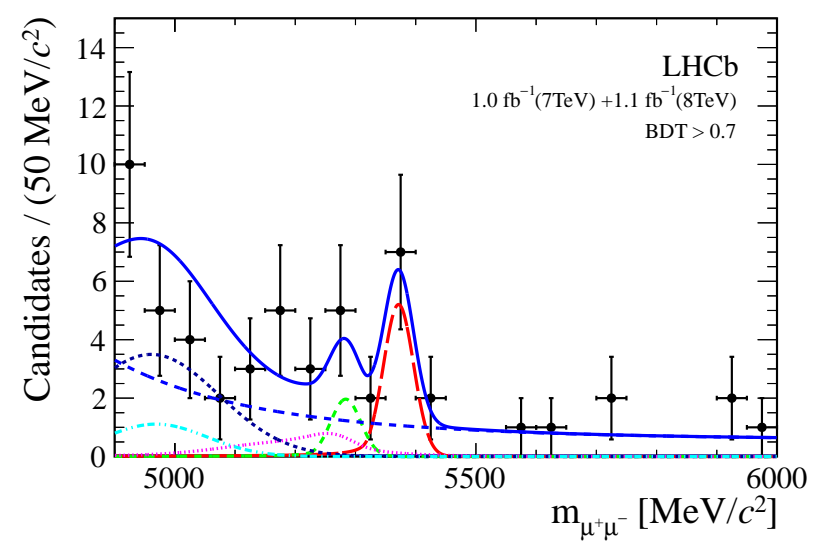

Figure 8: Invariant mass distribution of the selected $B_{s}^{0} \rightarrow$ $\mu^{+} \mu^{-}$candidates. The result of the fit is illustrated (blue solid line) with different components: $B_{s}^{0} \rightarrow \mu^{+} \mu^{-}$(red long-dashed line), $B^{0} \rightarrow \mu^{+} \mu^{-}$(green medium-dashed line), combinatorial background (blue medium-dashed line) and contamination from the other $B$ hadron decays (remaining lines). 
and $B^{0} \rightarrow \pi^{+} \pi^{-}$. The resolutions are estimated by combining the results obtained with a power-law interpolation between the measured resolutions of charmonium and bottomonium resonances decaying into two muons with those obtained with a fit of the mass distributions of control channels. The data in the $B_{s}^{0}$ search region show an excess of events with respect to the background expectation with a statistical significance of 3.5 standard deviations. It implies that such an excess of $B_{s}^{0} \rightarrow \mu^{+} \mu^{-}$ candidates is induced by the signal with probability of $1-5.3 \times 10^{-4}=0.99947$. By applying a maximumlikelihood fit to the data, the branching fraction is estimated to be $\mathcal{B}\left(B_{s}^{0} \rightarrow \mu^{+} \mu^{-}\right)=3.2_{-1.2}^{+1.5} \times 10^{-9}$, where the uncertainty is fully dominated by the statistical component. This is the first evidence for the decay $B_{s}^{0} \rightarrow \mu^{+} \mu^{-}$ and the measured branching fraction is in agreement with the Standard Model prediction. In the $B^{0}$ search region, the data are consistent with the background expectation. This result sets currently the world's best upper limit of $\mathcal{B}\left(B^{0} \rightarrow \mu^{+} \mu^{-}\right)<9.4 \times 10^{-10}$ at $95 \%$ confidence level.

\subsection{2 $B^{0} \rightarrow K^{* 0} \mu^{+} \mu^{-}$}

Another FCNC decay of high interest, $B^{0} \rightarrow K^{* 0} \mu^{+} \mu^{-}$, was recently studied with 2011 data sample corresponding to an integrated luminosity of $1.0 \mathrm{fb}^{-1}$ [6]. In this analysis, the differential branching fraction and a series of angular observables are investigated as a function of dimuon invariant mass squared, $q^{2}$. To select signal candidates among the triggered events a loose selection, based on the $B$ hadron decay time and daughter impact parameters, is applied at the first stage. A tight multivariate selection, using a boosted decision tree, is employed afterwards to suppress the remaining background. It allows to achieve a signal-to-background ratio in a $100 \mathrm{MeV} / c^{2}$ window around the reconstructed $B$ mass of about three-

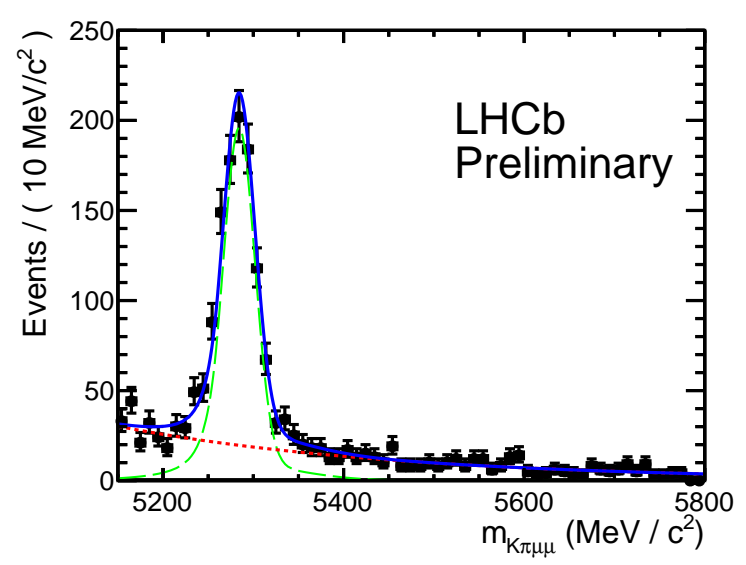

Figure 9: Invariant mass distribution of the selected $B^{0} \rightarrow$ $K^{* 0} \mu^{+} \mu^{-}$candidates in the range $4 m_{\mu}^{2}<q^{2}<19 \mathrm{GeV}^{2} / c^{4}$. The result of the fit is illustrated with the blue solid line, while the signal and background components are shown with the green long-dashed and red dashed lines, respectively.

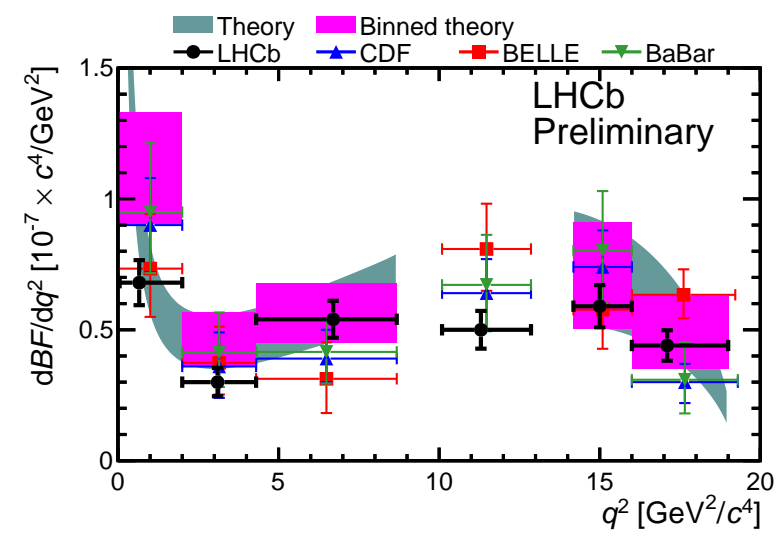

Figure 10: Differential branching fraction of the $B^{0} \rightarrow$ $K^{* 0} \mu^{+} \mu^{-}$decay as a function of $q^{2}$. Points include both statistical and systematic uncertainties. The BABAR [7], Belle [8] and CDF [9] measurements are shown too. The theory predictions are described in Ref. [10].
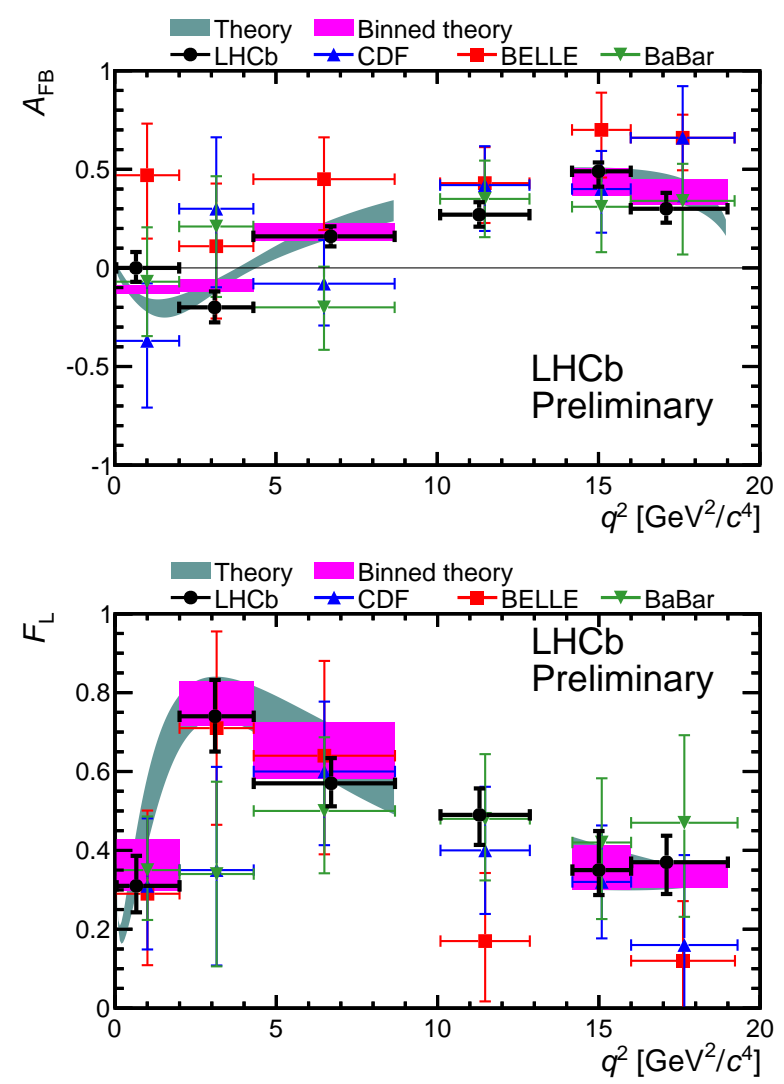

Figure 11: Forward-backward asymmetry of the dimuon system, $A_{\mathrm{FB}}$, (top) and fraction of longitudinal polarisation of the $K^{* 0}$ meson, $F_{\mathrm{L}}$, (bottom) as a function of $q^{2}$. Points include both statistical and systematic uncertainties. The BABAR [7], Belle [8] and CDF [9] measurements are shown too. The theory predictions are described in Ref. [10].

to-one. Specific peaking backgrounds from $B$ hadron decay channels with similar topology are suppressed using 
tight particle identification requirements and excluding the regions of dimuon mass around the $c \bar{c}$ resonances. The resulting invariant mass distribution of signal candidates in the range $4 m_{\mu}^{2}<q^{2}<19 \mathrm{GeV}^{2} / c^{4}$ is shown in Fig. 9, while Fig. 10 illustrates the observed differential branching fraction as a function of $q^{2}$. The latter indicates that the measurements are in good agreement with the Standard Model predictions. This is also the case for the angular observables of interest, some of which are shown in Fig. 11. A first measurement of the zero-crossing point of the forward-backward asymmetry of the dimuon system is additionally performed in this analysis. It is determined to be $q_{0}^{2}=\left(4.9_{-1.3}^{+1.1}\right) \mathrm{GeV}^{2} / c^{4}$, where the uncertainty is fully dominated by the statistical component. The results obtained in this study are the most precise to date.

\section{$3.2 C P$-violating phase $\phi_{\mathrm{s}}$}

Using currently available data, the $\mathrm{LHCb}$ collaboration has performed high-precision studies of $C P$ asymmetry in different $B$ hadron decay channels [11-21]. One of the most interesting analyses was conducted to measure the mixing-induced $C P$-violating phase $\phi_{\mathrm{s}}$. It can be determined without theoretical uncertainties using $B_{s}^{0}$ decays proceeding via the $b \rightarrow c \bar{c} s$ transition by measuring the time-dependent $C P$ asymmetry, which arises in this process due to the interference between the amplitudes of the transition with and without oscillation. In the Standard Model, the $\phi_{\mathrm{s}}$ phase is small and accurately predicted, while it may be significantly enlarged by New Physics. As a result, its measurement provides a sensitive test of the Standard Model.

For accurate measurement of the $\phi_{\mathrm{s}}$ phase the $\mathrm{LHCb}$ collaboration has performed a series of independent analyses using the following $B_{s}^{0}$ decay channels which occur via the $b \rightarrow c \bar{c} s$ transition: $B_{s}^{0} \rightarrow J / \psi f_{0}(980)$ [22], $B_{s}^{0} \rightarrow J / \psi \phi[23]$ and $B_{s}^{0} \rightarrow J / \psi \pi^{+} \pi^{-}$[24]. In these studies, signal candidates are selected exploiting typical features of $B$ hadron decays (long lifetime of $B$ hadrons, daughter particles with high $p_{\mathrm{T}}$ ) and imposing additional requirements on intermediate resonances and particle identification. The flavour tagging is based on properties of the decay of the other $B$ hadron in the event and has an efficiency times dilution-squared of about $2 \%$. The selected signal events are found to have an effective decay time resolution of better than $50 \mathrm{fs}$. The $\phi_{\mathrm{s}}$ phase is determined using a time-dependent fit to the data. The results obtained for the $B_{s}^{0} \rightarrow J / \psi \phi$ and $B_{s}^{0} \rightarrow J / \psi \pi^{+} \pi^{-}$decays are combined by performing a simultaneous fit to the data. It yields a value of $\phi_{\mathrm{s}}=-0.002 \pm 0.083 \pm 0.027 \mathrm{rad}$, where the first uncertainty is statistical and the second is systematic [23]. This result is the most precise to date and is consistent with the Standard Model expectation of $-0.0363_{-0.0015}^{+0.0016} \mathrm{rad}$.

\subsection{Evidence for $C P$ violation in the charm sector}

In addition to a rich $B$ physics programme, the $\mathrm{LHCb}$ collaboration aims to carry out high-precision measurements of all the key observables also in the charm sector. The latter is feasible due to the high production rate of open charm and excellent detector performance allowing the selection of high purity samples of hadronic and muonic $D$ meson decays. Studies of $D$ mesons offer, in particular, a unique opportunity to access up-type quarks in FCNC processes. The primary goal of the experiment in this area of research is to explore $C P$ violation and $D^{0}-\bar{D}^{0}$ mixing along with a search for rare $D$ meson decays.

Using currently available data, $\mathrm{LHCb}$ has carried out various measurements in the charm sector [25-36]. One of the most interesting results was obtained by searching time-integrated $C P$ violation in $D^{0} \rightarrow h^{+} h^{-}(h=K, \pi)$ decays using $0.62 \mathrm{fb}^{-1}$ of data recorded in 2011 [37]. In this analysis, the difference in $C P$ asymmetry between $D^{0} \rightarrow K^{+} K^{-}$and $D^{0} \rightarrow \pi^{+} \pi^{-}$decays is measured to be $\Delta A_{\mathrm{CP}}=(-0.82 \pm 0.21 \pm 0.11) \%$, where the first uncertainty is statistical and the second is systematic. This result differs from the hypothesis of $C P$ conservation by 3.5 standard deviations and thus, can be considered as the first evidence for $C P$ violation in the charm sector.

\subsection{Forward energy flow and charged particle multiplicities}

Besides an intensive heavy flavour physics programme, the $\mathrm{LHCb}$ experiment has performed important studies of QCD and electroweak processes in a unique kinematic range, which deliver valuable input to the knowledge of the parton density functions, underlying event activity, low Bjorken-x QCD dynamics and exclusive processes [3846]. Furthermore, some of these analyses have direct relevance to cosmic-ray and astroparticle physics. In particular, measurements of forward particle production in highenergy hadron-hadron collisions give an opportunity to impose strong constraints on cosmic-ray interaction models which are widely used in extensive air shower simulations. In these models, the primary particle production is dominated by forward and soft QCD interactions, which are described by parameters constrained mainly using previous collider data. At LHC collision energies, a more reliable determination of the cosmic-ray energy and composition becomes possible [47].

The first analysis where the LHCb data are compared to predictions given by cosmic-ray interaction models is the measurement of the energy flow $1 / N_{\text {int }} d E_{\text {total }} / d \eta$ created in $p p$ collisions at $\sqrt{s}=7 \mathrm{TeV}$ within the pseudorapidity range $1.9<\eta<4.9$ [48]. At large values of pseudorapidity this observable is expected to be directly sensitive to the amount of parton radiation and multi-parton interactions [49]. The latter represent a predominant contribution to the soft component of a hadron-hadron collision, called the underlying event. Its precise theoretical description still remains a challenge and this measurement is conducted to constrain the corresponding models. As described in Ref. [48], the primary measurement is the energy flow carried by charged particles, while a data-constrained Monte Carlo estimate of the neutral component is used for the measurement of the total energy flow. To probe various aspects of multi-particle production in hadron-hadron collisions, the measurements are 

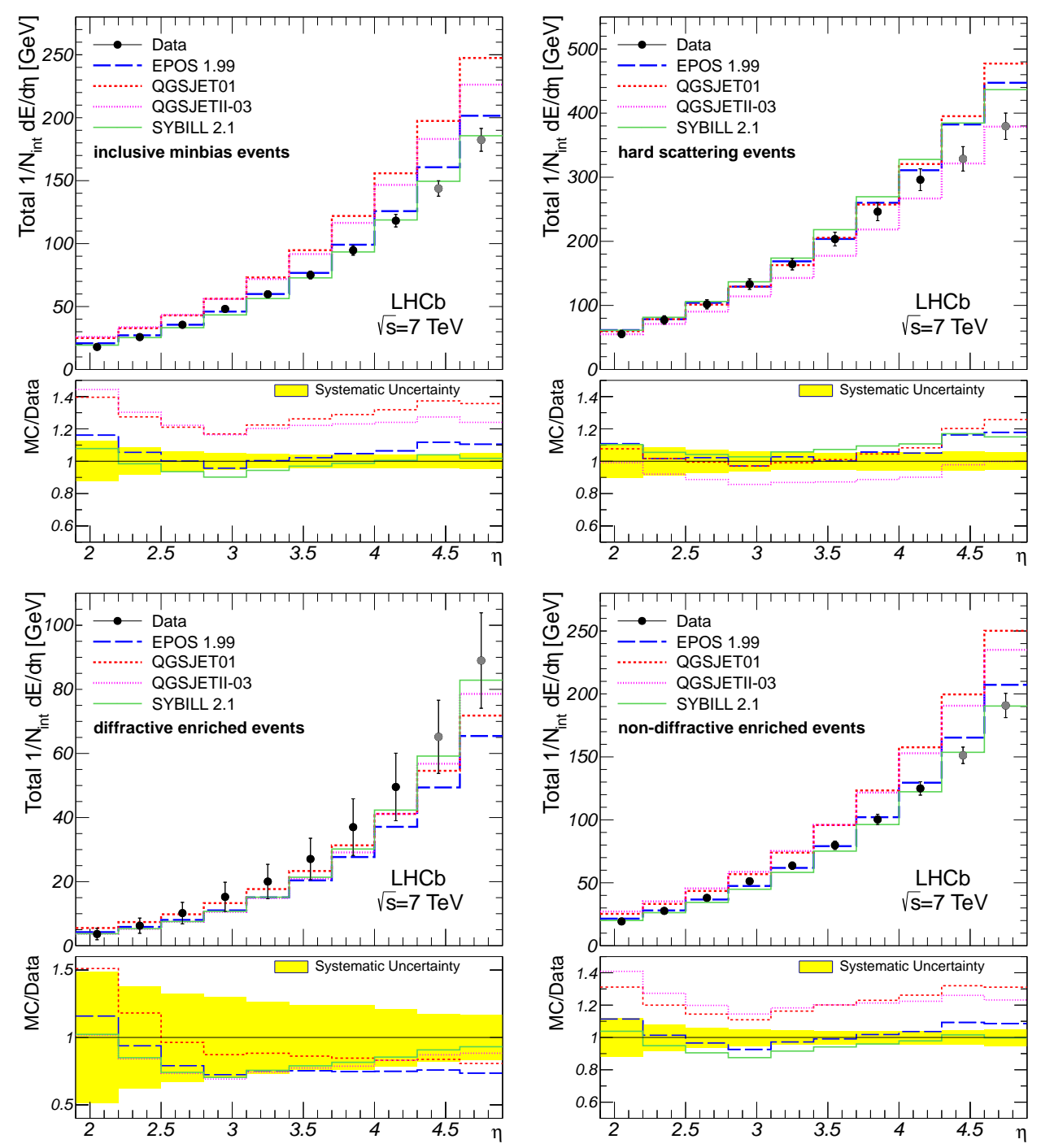

Figure 12: Total energy flow as a function of $\eta$ for all event classes as indicated in the figures. The corrected measurements are given by points with error bars, while the predictions by the cosmic-ray interaction models are shown as histograms. The comparison of the data against Pyтніa-based predictions can be found in Ref. [48]. The error bars represent the systematic uncertainties, which are highly correlated between the bins.

performed for inclusive minimum-bias (containing at least one track with $p>2 \mathrm{GeV} / \mathrm{c}$ in $1.9<\eta<4.9)$, hard scattering (having at least one track with $p_{\mathrm{T}}>3 \mathrm{GeV} / \mathrm{c}$ in $1.9<\eta<4.9)$, diffractive, and non-diffractive enriched interactions. The last two event types were selected among the inclusive minimum-bias interactions requiring the absence or presence of at least one backward track reconstructed by the VELO in $-3.5<\eta<-1.5$, respectively. In addition to cosmic-ray interaction models, experimental results are compared to predictions given by the PүтнIAbased event generators [50-52], which model the underlying event activity in different ways. Though the evolution of the energy flow as a function of $\eta$ is reasonably well reproduced by the Monte Carlo event generators, none of the models used in this analysis are able to describe the energy flow measurements for all event classes that have been studied. The majority of the РүтніA tunes underestimate the measurements at large $\eta$, while most of the cosmic-ray interaction models overestimate them as can be seen in Fig. 12. The energy flow is found to increase with the momentum transfer in an underlying $p p$ inelastic interaction.

Another $\mathrm{LHCb}$ analysis constraining the underlying event models being relevant for extensive air shower simulations is the measurement of charged particle production in $p p$ collisions at $\sqrt{s}=7 \mathrm{TeV}$ [53]. In this study, the multiplicity and density distributions of charged particle tracks originating from the primary interaction are investigated as a function of $\eta$ within the ranges $2.0<\eta<4.5$ and $-2.5<\eta<-2.0$ exploiting high detection efficiency of the VELO in these phase space regions. The measurements are carried out for events recorded with a minimum bias trigger requiring the presence of at least one reconstructed track in the VELO and for events containing at least one track with $p_{\mathrm{T}}>1 \mathrm{GeV} / c$. The results are compared to predictions given by several PYтHIA-based event 
generators [50-52]. None of these are able to describe fully the charged particle multiplicity and density distributions as a function of $\eta$. The considered PyтнIA tunes are found to underestimate the charged particle production in the phase space regions of the measurements.

\section{Summary}

The LHCb spectrometer possesses excellent tracking and vertexing performance, robust particle identification and flexible trigger system. The experiment has demonstrated the ability to take data of high quality successfully coping with extreme running conditions. Using data collected in the years 2010-2012, the LHCb collaboration has performed the world's most precise measurements of various important physics observables and has observed for the first time different rare processes in the heavy flavour sector. In addition, the experiment carries out important measurements of QCD and electroweak processes in a unique kinematic range. The physics potential of the experiment is expected to be fully exploited at higher LHC collision energy and interaction rate with an upgraded detector possessing enhanced trigger capabilities [54]. This would allow an order of magnitude more data to be collected and improving sensitivity to many important physics processes.

\section{References}

[1] LHCb Collaboration, JINST 3 (2008) S08005.

[2] LHCb Collaboration, Phys. Lett. B708 (2012) 241248.

[3] LHCb Collaboration, arXiv:1208.3355 [hep-ex].

[4] LHCb Collaboration, Phys. Rev. Lett. 110 (2013) 021801.

[5] R. E. Schapire, Y. Freund, Jour. Comp. and Syst. Sc. 55 (1997) 119.

[6] LHCb Collaboration, LHCb-CONF-2012-008.

[7] BABAR Collaboration, Phys. Rev. D79 (2009) 031102.

[8] Belle Collaboration, Phys. Rev. Lett. 103 (2009) 171801.

[9] CDF Collaboration, Phys. Rev. Lett. 108 (2012) 081807.

[10] C. Bobeth et al., JHEP 07 (2011) 067.

[11] LHCb Collaboration, Phys. Rev. Lett. 108 (2012) 201601.

[12] LHCb Collaboration, Phys. Rev. D85 (2012) 091105.

[13] LHCb Collaboration, Phys.Lett. B712 (2012) 203212.

[14] LHCb Collaboration, Nucl. Phys. B867 (2012) 1-18.

[15] LHCb Collaboration, Phys. Lett. B718 (2012) 43-55.
[16] LHCb Collaboration, arXiv:1210.4492 [hep-ex].

[17] LHCb Collaboration, arXiv:1211.6093 [hep-ex].

[18] LHCb Collaboration, arXiv:1212.5205 [hep-ex].

[19] LHCb Collaboration, LHCb-CONF-2012-018.

[20] LHCb Collaboration, LHCb-CONF-2012-022.

[21] LHCb Collaboration, LHCb-CONF-2012-028.

[22] LHCb Collaboration, Phys. Lett. B707 (2012) $497-$ 505.

[23] LHCb Collaboration, LHCb-CONF-2012-002.

[24] LHCb Collaboration, Phys. Lett. B713 (2012) 378386.

[25] LHCb Collaboration, Eur. Phys. J. C71 (2011) 1645.

[26] LHCb Collaboration, Phys. Lett. B707 (2012) 52.

[27] LHCb Collaboration, JHEP 04 (2012) 129.

[28] LHCb Collaboration, Phys. Lett. B714 (2012) 215223.

[29] LHCb Collaboration, Eur. Phys. J. C72 (2012) 2025.

[30] LHCb Collaboration, Phys. Lett. B718 (2012) 431440.

[31] LHCb Collaboration, Phys. Lett. B713 (2012) 186195.

[32] LHCb Collaboration, JHEP 06 (2012) 141.

[33] LHCb Collaboration, JHEP 10 (2012) 151.

[34] LHCb Collaboration, Phys. Lett. B718 (2013) $902-$ 909.

[35] LHCb Collaboration, arXiv:1211.1230 [hep-ex].

[36] LHCb Collaboration, arXiv:1212.1045 [hep-ex].

[37] LHCb Collaboration, Phys. Rev. Lett. 108 (2012) 111602.

[38] LHCb Collaboration, Phys. Lett. B693 (2010) 69-80.

[39] LHCb Collaboration, Phys. Lett. B703 (2011) $267-$ 273.

[40] LHCb Collaboration, JHEP 08 (2011) 034.

[41] LHCb Collaboration, Eur. Phys. J. C72 (2012) 2168.

[42] LHCb Collaboration, LHCb-CONF-2011-022.

[43] LHCb Collaboration, JHEP 06 (2012) 058.

[44] LHCb Collaboration, LHCb-CONF-2012-013.

[45] LHCb Collaboration, arXiv:1210.6289 [hep-ex].

[46] LHCb Collaboration, arXiv:1212.4620 [hep-ex].

[47] D. d'Enterria et al., Astropart.Phys. 35 (2011) 98113.

[48] LHCb Collaboration, arXiv:1212.4755 [hep-ex].

[49] T. Sjostrand, M. van Zijl, Phys. Rev. D36 (1987) 2019.

[50] P. Skands, Phys. Rev. D82 (2010) 074018.

[51] M. Clemencic et al., Journal of Physics: Conference Series 331 (2011), no. 3032023.

[52] T. Sjostrand et al., Comput. Phys. Commun. 178 (2008) 852.

[53] LHCb Collaboration, Eur. Phys. J. C72 (2012) 1947.

[54] LHCb Collaboration, CERN-LHCC-2012-007. 